J. Lake Sci. (湖泊科学) , 2012, 24(6): 932-942

http: //www. jlakes.org. E-mail : jlakes@niglas.ac.cn

(c) 2012 by Journal of Lake Sciences

\title{
大型浅水湖泊与大气之间的动量和水热交换系数——太湖为例"
}

\author{
肖 薇 ${ }^{1,2}$, 刘寿东 ${ }^{1,2}$, 李旭辉 ${ }^{1}$, 王 伟 ${ }^{1,2}$, 胡 凝 $^{2}$, 江晓东 ${ }^{2}$, 李永秀 ${ }^{2}$, 徐向华 ${ }^{2}$, 张雪松 ${ }^{2}$ \\ ( 1 : 南京信息工程大学大气环境中心, 南京 210044) \\ (2:南京信息工程大学应用气象学院,南京 210044)
}

\begin{abstract}
摘 要: 湖泊水面与大气之间垂直方向的动量、水汽和热量通量与风速、湿度和温度梯度之间存在比例关系, 因此在湖泊 水一气相互作用研究中, 这比例系数 (交换系数) 是关键因子. 在以往的研究中, 交换系数通常直接采用水面梯度观测法或 海洋大气近地层的参数化方案进行计算. 本文采用浴度相关系统和小气候系统仪器在太湖平台上直接观测的通量和气 象要素, 对上述交换系数 (最小均方差原则) 进行优化,结果为: 动量交换系数 $C_{\mathrm{D} 10 \mathrm{~N}}=1.52 \times 10^{-3}$ 、水汽交换系数 $C_{\mathrm{E} 10 \mathrm{~N}}=$ $0.82 \times 10^{-3}$ 、热量交换系数 $C_{\mathrm{H} 10 \mathrm{~N}}=1.02 \times 10^{-3}$, 与其他内陆湖泊浴度相关观测数据的推导结果一致. 本文的研究结果表 明: 与海洋参数化方案相比, 在相同的风速条件下, 湖面的空气动力学粗粘度比海洋高, 这可能是由于受到水深的影响; 如果采用海洋参数化方案, 会导致湖泊年蒸发量的估算值偏大 $40 \%$. 太湖的动量、水汽和热量交换系数可以视为常数, 可 以不考虑稳定度和风速的影响. 这是因为本文中 $83 \%$ 的数据为近中性条件. 敏感性分析表明: 如果考虑稳定度的影响, $L E$ 模拟值的平均误差降低了 $0.5 \mathrm{~W} / \mathrm{m}^{2}, H$ 的平均误差降低了 $0.4 \mathrm{~W} / \mathrm{m}^{2}, u_{*}$ 的计算值没有变化; 如果考虑风速的影响, $u_{*}$ 模 拟值的平均误差降低了 $0.004 \mathrm{~m} / \mathrm{s}, L E$ 的平均误差升高了 $1.3 \mathrm{~W} / \mathrm{m}^{2}, H$ 的模拟结果几乎不受影响. 这一结果能为湖气相 互作用研究提供参考.
\end{abstract}

关键词: 水一气交换系数;动量通量; 热量通量; 水汽通量;水面蒸发;太湖

\section{Transfer coefficients of momentum, heat and water vapour in the atmospheric surface layer of a large shallow freshwater lake: A case study of Lake Taihu}

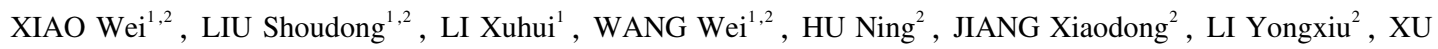
Xianghua $^{2} \&$ ZHANG Xuesong ${ }^{2}$

(1: Yale-NUIST Center on Atmospheric Environment, Nanjing University of Information Science and Technology, Nanjing 210044 , P. R. China)

(2: College of Applied Meteorology, Nanjing University of Information Science and Technology, Nanjing 210044, P. R. China)

Abstract: In studies of lake-atmosphere interactions, the fluxes of momentum, water vapor and heat (latent and sensible heat) are parameterized as being proportional to the differences in wind, humidity and air temperature between the water surface and a reference height above the surface. The proportionality or transfer coefficients are often assumed to follow the gradient observation above lake surface or the parameterizations established for the marine atmospheric surface layer. Optimization against the eddy covariance and micrometeorology measurements made over a large shallow freshwater lake (Lake Taihu) shows that the transfer coefficients of momentum $\left(C_{\mathrm{D} 10 \mathrm{~N}}\right)$, water $\left(C_{\mathrm{E} 10 \mathrm{~N}}\right)$ and heat $\left(C_{\mathrm{H} 10 \mathrm{~N}}\right)$ were $C_{\mathrm{D} 10 \mathrm{~N}}=1.52 \times 10^{-3}, C_{\mathrm{E} 10 \mathrm{~N}}=0.82 \times 10^{-3}$ and $C_{\mathrm{H} 10 \mathrm{~N}}=1.02 \times$ $10^{-3}$, respectively. These values are in good agreement with the values derived from the eddy covariance measurement in other inland lakes. Comparison with oceanographic parameterizations suggests that lake surfaces were aerodynamically rougher than open oceans under similar wind conditions, which may due to the shallow depth of the lake, and that these parameterizations can bias the annual lake evaporation estimate by as much as $40 \%$ higher. Our results also suggest that these coefficients can be regarded as constants independent of stability and wind speed. Sensitivity analysis indicated that the mean error between calculation and observa-

* 江苏省基础研究计划 (自然科学基金) 面上研究项目 (BK2011830)、教育部长江学者和创新团队发展计划项目 (PCSIRT) 和江苏高校优势学科建设工程项目 (PAPD) 联合资助. 2012-04-08 收稿; 2012-05-30 收修改稿. 肖 薇,女,1982 年生,博士,讲师;E-mail: wei. xiao@ nuist. edu. cn. 
tion of latent heat flux $(L E)$ decreased $0.5 \mathrm{~W} / \mathrm{m}^{2}$, that of sensible heat flux $(H)$ decreased $0.4 \mathrm{~W} / \mathrm{m}^{2}$, and no difference between friction velocity $\left(u_{*}\right)$ calculation and observation if stability correction was considered since around $83 \%$ of data were in neutral condition. If the effect of wind was considered, the mean error between calculation and observation decreased $0.004 \mathrm{~m} / \mathrm{s}$ for $u_{*}$, increased $1.3 \mathrm{~W} / \mathrm{m}^{2}$ for $L E$, and negligible for $H$. This study can provide reference for the research on lake-atmosphere interaction.

Keywords: Water-atmosphere exchange coefficients; momentum flux; heat flux; water vapor flux; evaporation; Lake Taihu

湖泊对大气有重要的影响. 全球有 $3.04 \times 10^{8}$ 个湖泊, 总面积为 $4.20 \times 10^{6} \mathrm{~km}^{2[1]}$. 与陆地相比, 湖泊的反 照率更低 ${ }^{[2-5]}$ 、热容量更大, 因此湖泊能储存更多的太阳辐射, 对气温变化有缓冲作用 ${ }^{[6-8]}$. 与陆地植被表面 相比, 湖泊更加平滑, 这会影响局地大气环流的变化 ${ }^{[9-10]}$. 另外, 湖泊对局地和全球尺度上的气候和天气有重 要影响 ${ }^{[3,8,11-12]}$.

陆地 (湖泊) 和大气之间的动量、感热和水汽通量是陆 (湖)气相互作用的主要驱动因子. 在陆地与大气 相互作用的研究中, 浴度相关方法被广泛采用, 直接测量陆地生态系统中的动量、感热和水汽通量, 但在湖 气相互作用研究中很少采用该方法 ${ }^{[13-16]}$, 主要原因是涡度相关系统仪器需要长期稳定的观测平台, 这在水 面上很难实现. 而更普遍的方法是采用传输方程计算上述通量:

$$
\begin{gathered}
\tau=\rho_{\mathrm{a}} C_{\mathrm{D}} u^{2} \\
L E=\rho_{\mathrm{a}} L_{\mathrm{v}} C_{\mathrm{E}} u\left(q_{\mathrm{s}}-q_{\mathrm{a}}\right) \\
H=\rho_{\mathrm{a}} c_{\mathrm{p}} C_{\mathrm{H}} u\left(\theta_{\mathrm{s}}-\theta_{\mathrm{a}}\right)
\end{gathered}
$$

式中, $\tau$ 为垂直动量通量 $\left(\mathrm{kg} /\left(\mathrm{s}^{2} \cdot \mathrm{m}\right)\right), L E$ 为潜热通量 $\left(\mathrm{W} / \mathrm{m}^{2}\right), H$ 为感热通量 $\left(\mathrm{W} / \mathrm{m}^{2}\right), \rho_{\mathrm{a}}$ 为空气密度 $\left(\mathrm{kg} / \mathrm{m}^{3}\right), u$ 为参考高度的风速 $(\mathrm{m} / \mathrm{s}), q_{\mathrm{s}}$ 和 $q_{\mathrm{a}}$ 为水面和参考高度的比湿 $(\mathrm{kg} / \mathrm{kg}), \theta_{\mathrm{s}}$ 和 $\theta_{\mathrm{a}}$ 为水面和参考高度 的位温 $(\mathrm{K}), C_{\mathrm{D}} 、 C_{\mathrm{E}}$ 和 $C_{\mathrm{H}}$ 分别为动量、水汽和感热交换系数 ${ }^{[16-25]}$. 从上述公式可以看出, 交换系数取值的偏 差会直接导致通量计算值的偏差,因此精确估算交换系数是至关重要的.

在数值天气预报和气候模型中加入湖泊模块会提高模型性能 ${ }^{[3,8,26]}$. 在这些模型中, 动量、感热和水汽 通量是气流和水热收支的下边界条件, 根据公式 $(1 \sim 3)$ 进行参数化 ${ }^{[2]}$, 交换系数从海洋试验观测中取 值 ${ }^{[18]}$. 但是目前还不确定海洋试验得到的交换系数是否适用于湖泊环境. 海洋和湖泊的环境条件有所不同, 例如浪高是影响动量交换系数的关键因子, 湖泊 (尤其是浅水湖泊) 中的浪高主要受湖泊深度的限制, 水深 是控制湖泊与大气之间相互作用的主要因子 ${ }^{[27]}$, 而海洋中的浪高则主要取决于风浪吹程 ${ }^{[28-33]}$. 另外, 海面风 速通常比内陆湖泊高得多. 有一些湖泊试验研究得到了交换系数 ${ }^{[5,13,34-36]}$, 但目前还没有发现直接采用通量 观测资料评价海洋参数化方案计算的 $C_{\mathrm{D}} 、 C_{\mathrm{E}}$ 和 $C_{\mathrm{H}}$ 在湖泊中适用性的文献.

最近, 本研究组在太湖安装了浴度相关系统仪器, 实地连续观测湖泊与大气之间的动量、热量和水汽通 量. 本文的目的是用通量观测资料评价多种计算交换系数参数化方案, 并且检验浅水湖泊环境中质量传输 方程对稳定度校正和风速的敏感性.

\section{1 研究方法}

\section{1 站点和仪器}

本研究的试验站点为中国科学院太湖湖泊生态系统研究站 (TLLER), 该站位于太湖北部梅梁湾内 $\left(31^{\circ} 24^{\prime} \mathrm{N}, 120^{\circ} 13^{\prime} \mathrm{E}\right)$. 太湖是中国第三大淡水湖泊,面积为 $2338 \mathrm{~km}^{2}$, 平均深度为 $1.9 \mathrm{~m}$.

浴度相关系统仪器架设在太湖中的水泥桩上, 距离岸边约 $100 \mathrm{~m}$. 分别采用三维超声风速仪 (CSAT3, Campbell Scientific Inc., Logan, UT, USA) 和开路式红外气体分析仪 (LI 7500, Li-Cor Inc, Lincoln, NE, USA) 测量风速和大气的 $\mathrm{H}_{2} \mathrm{O} / \mathrm{CO}_{2}$ 浓度, 采样频率为 $10 \mathrm{~Hz}$. 垂直动量通量 $(\tau)$ 、感热通量 $(H)$ 和潜热通量 $(L E)$ 输出值的时间步长为 $30 \mathrm{~min}$. 观测高度距离湖面 $3.5 \mathrm{~m}$. 用净辐射仪 (CNR4, Kipp \& Zonen B. V., Delft, The Netherlands) 观测辐射平衡的四个组分(人射太阳辐射、反射短波辐射、 湖面长波辐射和大气逆辐射). 
采用小气候系统仪器 (Dynakmet, Dynamax Inc., Houston, TX, USA) 观测气温、相对湿度、风速、风向, 观测高度距离湖面 $4.0 \mathrm{~m}$. 用水温计 (109-L, Campbell Scientific Inc.) 测量 20、50、100 和 $150 \mathrm{~cm}$ 水深处的水 温和底泥温度.

浴度相关系统仪器和小气候系统仪器于 2010 年 6 月 14 日安装并开始观测. 本文采用 2010 年 6 月 14 日一 12 月 31 日风向 $200^{\circ} \sim 300^{\circ}$ 的观测数据. 在这个风向范围内, 超声风速仪受到仪器支架的干扰最小. 在 这个研究时段内, $3.5 \mathrm{~m}$ 高度处的风速平均值为 $3.8 \mathrm{~m} / \mathrm{s}$, 变化范围为 $0.2 \sim 12.8 \mathrm{~m} / \mathrm{s}$; 气温的平均值为 $20.5^{\circ} \mathrm{C}$, 变化范围为 $-2.2 \sim 36.6^{\circ} \mathrm{C}$; 水温 $\left(20 \mathrm{~cm}\right.$ 深度) 的平均值为 $21.5^{\circ} \mathrm{C}$, 变化范围为 $0.9 \sim 35.9^{\circ} \mathrm{C}$; 气温 和水温差值的平均值为 $-1.1^{\circ} \mathrm{C}$, 变化范围为 $-6.6 \sim 5.8^{\circ} \mathrm{C} .20 \mathrm{~cm}$ 水深处的水温 $\left(T_{\mathrm{w}}\right)$ 与水面热辐射温度 $\left(T_{\mathrm{s}}\right)$ 的总体变化趋势一致性较好, $R^{2}=0.99$, 但是夏季和冬季的日变化幅度明显不同, 夏季白天 $T_{\mathrm{w}}<T_{\mathrm{s}}$ 、夜 间 $T_{\mathrm{w}}>T_{\mathrm{s}}$, 而冬季 $T_{\mathrm{w}}<T_{\mathrm{s}}$ 的情况仅在凌晨出现. 风浪吹程大于 $8 \mathrm{~km} .83 \%$ 的数据为近中性层结条件 $(|\zeta|<0.1)$.

\section{2 资料分析}

根据公式( $1 \sim 3)$, 用 $\tau 、 L E$ 和 $H$ 观测值和风速、湿度和温度观测值, 对交换系数 $C_{\mathrm{D}} 、 C_{\mathrm{E}}$ 和 $C_{\mathrm{H}}$ 进行优化. 其中水面温度由水面长波辐射通量观测值和斯蒂芬-玻尔兹曼定律计算得到, 发射率取值为 0.97 . 在参数优 化中, 不考虑稳定度的影响, 将优化结果直接表示为中性稳定度条件的交换系数, 用下标 $\mathrm{N}$ 表示. 交换系数 与水表粗糙度之间的关系为:

$$
\begin{gathered}
C_{\mathrm{DN}}=k^{2} /\left[\ln \left(z / z_{0}\right)\right]^{2} \\
C_{\mathrm{EN}}=k^{2} /\left[\ln \left(z / z_{0}\right) \ln \left(z / z_{\mathrm{q}}\right)\right] \\
C_{\mathrm{HN}}=k^{2} /\left[\ln \left(z / z_{0}\right) \ln \left(z / z_{\mathrm{T}}\right)\right]
\end{gathered}
$$

式中, $z_{0} 、 z_{\mathrm{q}}$ 和 $z_{\mathrm{T}}$ 分别为动量、水汽和热量粗粩度 $(\mathrm{m}), k$ 是 von Karman 常数, $z=3.5 \mathrm{~m}$, 是参考高度 ( 风速的观 测高度). 为了方便与相关文献进行对比, 将交换系数换算成水面以上 $10 \mathrm{~m}$ 处的交换系数, 公式分别为 $C_{\mathrm{D} 10 \mathrm{~N}} 、 C_{\mathrm{E} 10 \mathrm{~N}}$ 和 $C_{\mathrm{H} 10 \mathrm{~N}}$ :

$$
\begin{gathered}
C_{\mathrm{D} 10 \mathrm{~N}}=k^{2} /\left[\ln \left(10 / z_{0}\right)\right]^{2} \\
C_{\mathrm{E} 10 \mathrm{~N}}=k^{2} /\left[\ln \left(10 / z_{0}\right) \ln \left(10 / z_{\mathrm{q}}\right)\right] \\
C_{\mathrm{HION}}=k^{2} /\left[\ln \left(10 / z_{0}\right) \ln \left(10 / z_{\mathrm{T}}\right)\right]
\end{gathered}
$$

式中, $z_{0} 、 z_{\mathrm{q}}$ 和 $z_{\mathrm{T}}$ 根据公式 $(4 \sim 6)$ 通过 $C_{\mathrm{DN}} 、 C_{\mathrm{EN}}$ 和 $C_{\mathrm{HN}}$ 的优化值计算得到.

\section{2 结果与讨论}

\section{1 交换系数}

2.1 .1 湖泊交换系数 本文采用研究时段的所有通量观测值对交换系数进行优化, 得到 $10 \mathrm{~m}$ 高度处的中性 交换系数为 $C_{\mathrm{D} 10 \mathrm{~N}}=1.52 \times 10^{-3} 、 C_{\mathrm{E} 10 \mathrm{~N}}=0.82 \times 10^{-3} 、 C_{\mathrm{H} 10 \mathrm{~N}}=1.02 \times 10^{-3}$, 对应的粗糙度为 $z_{0}=3.5 \times 10^{-4} \mathrm{~m}$ 、 $z_{\mathrm{q}}=6.0 \times 10^{-8} \mathrm{~m} 、 z_{\mathrm{T}}=2.4 \times 10^{-6} \mathrm{~m}$. 如果采用每半小时的观测值计算交换系数, 得到 $C_{\mathrm{D} 10 \mathrm{~N}} 、 C_{\mathrm{E} 10 \mathrm{~N}}$ 和 $C_{\mathrm{H} 10 \mathrm{~N}}$ 的标 准差分别为 $5.78 \times 10^{-3} 、 0.62 \times 10^{-3}$ 和 $8.78 \times 10^{-3}$, 离散度非常大, 这是因为当通量很小的时候, 相应的风 速、温度和湿度的梯度也非常小, 用公式 (1 3) 计算时, 分母太小, 就会产生很大偏差. 因此, 采用全部数据 进行参数化比较合理,但我们也无法确定交换系数对风速的依赖性.

为了与其他淡水湖泊的交换系数做比较, 我们选取了 4 个有通量观测的淡水湖泊, 分别为 Lake ValkeaKotinen $^{[36]}$ 、Lake Tämnaren ${ }^{[34]}$ 、Ross Rarnett Reservoir ${ }^{[16]}$ 和 Great Slave Lake ${ }^{[35]}$, 根据公式 $(1 \sim 3)$, 推导交换系 数得出 $C_{\mathrm{D} 10 \mathrm{~N}}$ 的平均值为 $1.55 \times 10^{-3}\left(1.21 \times 10^{-3} \sim 1.89 \times 10^{-3}\right), C_{\mathrm{E} 10 \mathrm{~N}}$ 的平均值为 $1.09 \times 10^{-3}\left(0.88 \times 10^{-3} \sim\right.$ $\left.1.44 \times 10^{-3}\right), C_{\mathrm{H} 10 \mathrm{~N}}$ 的平均值为 $1.03 \times 10^{-3}\left(0.49 \times 10^{-3} \sim 1.25 \times 10^{-3}\right)$, 比太湖的 $C_{\mathrm{D} 10 \mathrm{~N}} 、 C_{\mathrm{E} 10 \mathrm{~N}}$ 和 $C_{\mathrm{H} 10 \mathrm{~N}}$ 值分别 高 $6.7 \% 、 37.5 \%$ 和 $0.01 \%$. 综合太湖和这 4 个湖泊的研究, 我们得到湖泊的交换系数平均值 (标准差) 为: $C_{\mathrm{DION}}=(1.54 \pm 0.26) \times 10^{-3} 、 C_{\mathrm{E} 10 \mathrm{~N}}=(1.03 \pm 0.24) \times 10^{-3}$ 和 $C_{\mathrm{HION}}=(1.02 \pm 0.31) \times 10^{-3}$ (观测点 $\left.n=5\right)$. 这 与水面梯度观测法得到的结果接近, 如抚仙湖的 $C_{\mathrm{D} 10 \mathrm{~N}}$ 为 $1.83 \times 10^{-3}$. 
上述 5 个湖泊的 $C_{\mathrm{E} 10 \mathrm{~N}}$ 和 $C_{\mathrm{H} 10 \mathrm{~N}}$ 估算值的误差可能是由水面温度计算误差引起的. 辐射计观测水面长波辐 射的不确定度为 $5 \sim 10 \mathrm{~W} / \mathrm{m}^{2}$, 导致 $T_{\mathrm{s}}$ 估算值的不确定度为 $2^{\circ} \mathrm{C}$. 如果 $T_{\mathrm{s}}$ 偏低 (偏高) $2^{\circ} \mathrm{C}$, 本文中 $C_{\mathrm{E} 10 \mathrm{~N}} 、 C_{\mathrm{H} 10 \mathrm{~N}}$ 的优化值分别为 $1.11 \times 10^{-3}\left(0.62 \times 10^{-3}\right) 、 3.87 \times 10^{-3}\left(0.55 \times 10^{-3}\right)$. 因此, 我们认为 $T_{\mathrm{s}}$ 误差是各文献中 $C_{\mathrm{E} 10 \mathrm{~N}}$ 和 $C_{\mathrm{H} 10 \mathrm{~N}}$ 估算值 (表 1$)$ 离散度大的原因之一. $C_{\mathrm{D} 10 \mathrm{~N}}$ 的估算不受此影响.

表 1 根据湖泊浴度相关观测数据计算的动量、水汽和热量交换系数

Tab. 1 A summary of transfer coefficients for momentum, moisture and heat calculated from the eddy covariance measurements in lakes

\begin{tabular}{|c|c|c|c|c|c|c|c|}
\hline 湖泊名称及位置 & $\begin{array}{l}\text { 面积/ } \\
\mathrm{km}^{2}\end{array}$ & $\begin{array}{c}\text { 平均深度 } \\
\mathrm{m}\end{array}$ & $\begin{array}{c}\text { 最大深度/ } \\
\text { m }\end{array}$ & 研究时段 & $\begin{array}{c}C_{\mathrm{D} 10 \mathrm{~N}} / \\
\left(\times 10^{3}\right)\end{array}$ & $\begin{array}{l}C_{\mathrm{E} 10 \mathrm{~N}} / \\
\left(\times 10^{3}\right)\end{array}$ & $\begin{array}{l}C_{\mathrm{H} 10 \mathrm{~N}} / \\
\left(\times 10^{3}\right)\end{array}$ \\
\hline $\begin{array}{l}\text { 中国太湖 } \\
\left(31^{\circ} 24^{\prime} \mathrm{N}, 120^{\circ} 13^{\prime} \mathrm{E}\right)\end{array}$ & 2338 & 1.9 & 4.9 & $2010-06-12-12-31$ & 1.52 & 0.82 & 1.02 \\
\hline $\begin{array}{l}\text { 芬兰 Lake Valkea-Kotinen } \\
\left(61^{\circ} 14^{\prime} \mathrm{N}, 25^{\circ} 3^{\prime} \mathrm{E}\right)\end{array}$ & 0.4 & 2.5 & 6.5 & $2005-2008$ 年 $4-10$ 月 & 1.21 & 1.06 & 1.25 \\
\hline $\begin{array}{l}\text { 瑞典 Lake Tämnaren } \\
\left(60^{\circ} 0^{\prime} \mathrm{N}, 12^{\circ} 12^{\prime} \mathrm{E}\right)\end{array}$ & 37 & 1.2 & 2. 0 & $\begin{array}{l}1994-06-08-06-23 \\
1995-06-20-07-14\end{array}$ & 1.42 & 0.88 & 1.13 \\
\hline $\begin{array}{l}\text { 美国 Ross Rarnett Reservoir } \\
\left(32^{\circ} 26^{\prime} \mathrm{N}, 90^{\circ} 2^{\prime} \mathrm{W}\right)\end{array}$ & 134 & 5.0 & 8. 0 & $\begin{array}{l}2007-09-01- \\
2008-02-28\end{array}$ & 1.89 & 0.97 & 1.23 \\
\hline $\begin{array}{l}\text { 加拿大 Great Slave Lake } \\
\left(61^{\circ} 55^{\prime} \mathrm{N}, 113^{\circ} 44^{\prime} \mathrm{W}\right)\end{array}$ & 27000 & 41.0 & 614.0 & 1997－1998 年无冰期 & 1.66 & 1.44 & 0.49 \\
\hline
\end{tabular}

$T_{\mathrm{w}}$ 和 $T_{\mathrm{s}}$ 观测值的对比结果表明, $20 \mathrm{~cm}$ 水深处的水温 $\left(T_{\mathrm{w}}\right)$ 与水面热辐射温度 $\left(T_{\mathrm{s}}\right)$ 的总体变化趋势一致 性较好, 相关系数达到 0.99 , 平均偏差为 $T_{\mathrm{s}}-T_{\mathrm{w}}=1.1^{\circ} \mathrm{C}$. 各个季节普遍的特征是正午水温偏低, 凌晨水温偏 高; 但是夏季水温偏高的时段较长, $T_{\mathrm{s}}-T_{\mathrm{w}}$ 最低值为 $-6.6^{\circ} \mathrm{C}$; 冬季水温偏低的时段较长, $T_{\mathrm{s}}-T_{\mathrm{w}}$ 最高值为 $5.8^{\circ} \mathrm{C}$.

本文中, $T_{\mathrm{s}}$ 的不确定性小于 $2^{\circ} \mathrm{C}$. 可以通过 $\left(T_{\mathrm{s}}-T_{\mathrm{a}}\right)$ 与 $H$ 的关系 (公式 3) 估算 $T_{\mathrm{s}}$ 的不确定性. 如果 $T_{\mathrm{s}}$ 的 观测是完全准确的, 那么 $\left(T_{\mathrm{s}}-T_{\mathrm{a}}\right)$ 与 $H$ 的线性回归方程的截距为 0 , 而水面上的大气近地层不会出现热量 传输的逆梯度. 本文的资料回归的截距为 $0.1025 \pm 0.0047^{\circ} \mathrm{C}$ (参数置信区间为 $95 \%$ ).

2.1 .2 海洋参数化方案结果 本文以太湖的观测数据为输人变量, 采用常用的海洋参数化方案 (附录 1 ) 计 算交换系数,结果见表 2. 输人变量为水面摩擦速度 $u_{*}(\mathrm{~m} / \mathrm{s})$ 和 $/$ 或 $10 \mathrm{~m}$ 高度处的风速 $u_{10}(\mathrm{~m} / \mathrm{s}) . u_{*}$ 为涡度 相关系统观测值, $u_{10}$ 由超声风速仪测量的 $3.5 \mathrm{~m}$ 高度风速外推得到, 计算公式为:

$$
u_{10} / u=\ln \left(10 / z_{0}\right) / \ln \left(3.5 / z_{0}\right)
$$

式中, $z_{0}$ 通过公式 (4)算得.

$\mathrm{BDY}$ 模式计算的 $C_{\mathrm{D} 10 \mathrm{~N}}$ 和 $C_{\mathrm{E} 10 \mathrm{~N}}$ 明显偏大, 在热带和中纬度海洋的湍流通量观测研究中, 也发现 BDY 模 式的结果不合理 ${ }^{[52]}$. 而其他 8 个参数化方案得到的结果比较一致: $C_{\mathrm{D} 10 \mathrm{~N}}=(1.21 \pm 0.14) \times 10^{-3} 、 C_{\mathrm{E} 10 \mathrm{~N}}=$ $(1.14 \pm 0.11) \times 10^{-3}$ 和 $C_{\mathrm{H} 10 \mathrm{~N}}=(1.22 \pm 0.39) \times 10^{-3} . T$ 检验结果表明, 海洋 $C_{\mathrm{D} 10 \mathrm{~N}}$ 和 $C_{\mathrm{E} 10 \mathrm{~N}}$ 平均值与湖泊的结 果存在明显差异, 置信度分别为 $P<0.01$ 和 $P<0.05$. 因此, 相同风速条件下, 用通量观测值反推的浅水湖泊 拖电系数明显比海洋参数化方案计算值偏高.

\section{2 动量、水汽和热量通量计算结果}

为了评估交换系数的可靠性, 根据公式 $(1 \sim 3)$ (不考虑稳定度校正, $C_{\mathrm{D}}=C_{\mathrm{D} 10 \mathrm{~N}} 、 C_{\mathrm{E}}=C_{\mathrm{E} 10 \mathrm{~N}}$ 和 $C_{\mathrm{H}}=$ $C_{\mathrm{H} 10 \mathrm{~N}}$ ), 分别采用湖泊交换系数优化结果的平均值和海洋参数化方案 (除 BDY) 计算结果的平均值计算太湖 的 $u_{*} 、 L E$ 和 $H$, 对比结果如图 1 (第 364 365 d) 、图 2(第 $276 \sim 277 \mathrm{~d}$, 第 $364 \sim 365 \mathrm{~d}$ ) 和表 3(第 $364 \sim 365 \mathrm{~d}$ ). 从较长时间尺度来看, 采用太湖交换系数的优化值计算通量, 总体效果明显优于海洋参数化方案的计算结 果 (图 1). 尽管在小时到日尺度上, 通量计算结果会存在系统偏差, 如图 2 中 $u_{*}$ 和 $L E$ 在第 $276 \sim 277 \mathrm{~d}$ 模拟 值偏高, 第 $364 \sim 365 \mathrm{~d}$ 模拟值偏低, 这是因为前一个时段风速平均值为 $4.8 \mathrm{~m} / \mathrm{s}$, 后一时段风速平均值为 $8.1 \mathrm{~m} / \mathrm{s}$, 实际的交换系数应该是前者低、后者高. 而本文中交换系数取常数, 导致第 $276 \sim 277 \mathrm{~d}$ 交换系数取 
值比实际值偏大, 计算出的 $u_{*}$ 和 $L E$ 比观测值系统偏高; 而第 $364 \sim 365 \mathrm{~d}$ 的情况则相反. 采用太湖交换系数 优化值, $u_{*} 、 L E$ 和 $H$ 观测值和模拟值的 $R^{2}$ 分别为 $0.69 、 0.62$ 和 0.77 (表 3 ). 采用海洋参数化方案结果, $u_{*}$ 和 $L E$ 的观测值与模拟值误差变大, $H$ 不受影响. $u_{*}$ 和 $L E$ 的平均偏差分别为 $-0.032 \mathrm{~m} / \mathrm{s}$ 和 $23.1 \mathrm{~W} / \mathrm{m}^{2}$, 分 别占 $u_{*}$ 和 $L E$ 观测值的 $17.8 \%$ 和 $43.4 \% . L E$ 估算的偏差会导致水量收支估算的偏差. 如果采用海洋参数方 案, $L E$ 的估算值偏高 $23.1 \mathrm{~W} / \mathrm{m}^{2}$, 太湖年蒸发量估算值偏差会达到 $300 \mathrm{~mm}$; 而根据太湖水文站蒸发池的观 测结果 ${ }^{[53-55]}$, 太湖年蒸发量的多年平均值约为 $980 \mathrm{~mm}$. 因此, 准确估算交换系数对于湖泊水量和能量收支的 研究至关重要. 另外, 这一结果表明无论是计算长期 (季节尺度, 图 1) 还是短期 (小时尺度, 图 2) 的湖气交换 过程, 质量传输方程 (公式 $1 \sim 3$ ) 是非常好的方法 ${ }^{[20,23,56]}$.

表 2 用太湖的观测资料和海洋参数化方法计算的交换系数平均值 (变化范围) *

Tab. 2 The mean values (ranges) of the transfer coefficients calculated with the measurement at Lake Taihu and the ocean parameterizations

\begin{tabular}{llllc}
\hline 海洋参数化方法 & 参考文献 & $C_{\mathrm{D} 10 \mathrm{~N}} /\left(\times 10^{-3}\right)$ & $C_{\mathrm{E} 10 \mathrm{~N}} /\left(\times 10^{-3}\right)$ & $C_{\mathrm{H} 10 \mathrm{~N}} /\left(\times 10^{-3}\right)$ \\
\hline BDY-NC & {$[37-40]$} & $3.89(1.01 \sim 248.21)$ & $1.72(1.19 \sim 13.5)$ & 0.70 \\
CAM3 & {$[22,41-42]$} & $1.46(1.05 \sim 12.57)$ & $1.29(1.12 \sim 3.88)$ & $1.22(1.06 \sim 3.67)$ \\
COARE 3.0 & {$[43-44]$} & $1.08(0.97 \sim 1.63)$ & $0.96(0.91 \sim 1.16)$ & $0.96(0.91 \sim 1.16)$ \\
ECMWF & {$[45-46]$} & $1.11(0.91 \sim 1.73)$ & $1.11(1.07 \sim 1.33)$ & $1.07(1.03 \sim 1.27)$ \\
GEOS-2 & {$[41,47]$} & $1.15(0.96 \sim 1.56)$ & $1.02(0.90 \sim 1.24)$ & $1.08(0.93 \sim 1.30)$ \\
GSSTF-2 & {$[48]$} & $1.11(0.99 \sim 1.65)$ & $1.12(1.06 \sim 1.33)$ & $1.02(1.02 \sim 1.26)$ \\
HOAPS & {$[49]$} & $1.32(1.25 \sim 1.90)$ & 1.20 & 1.00 \\
J-OFURO & {$[41,47,50]$} & $1.14(1.14 \sim 1.43)$ & $1.19(1.08 \sim 1.57)$ & $2.16(1.97 \sim 2.85)$ \\
UA & {$[20,49,51]$} & $1.32(1.25 \sim 1.90)$ & $1.26(1.19 \sim 1.86)$ & $1.26(1.19 \sim 1.86)$ \\
\hline
\end{tabular}

* 几种海洋参数化方法 (除 $\mathrm{BDY}$ 方案以外) 计算的 $C_{\mathrm{D} 10 \mathrm{~N}} 、 C_{\mathrm{E} 10 \mathrm{~N}}$ 和 $C_{\mathrm{H} 10 \mathrm{~N}}$ 的平均值 (变化范围) 分别是 $(1.21 \pm 0.14) \times$ $10^{-3} 、(1.14 \pm 0.11) \times 10^{-3}$ 和 $(1.22 \pm 0.39) \times 10^{-3}$.
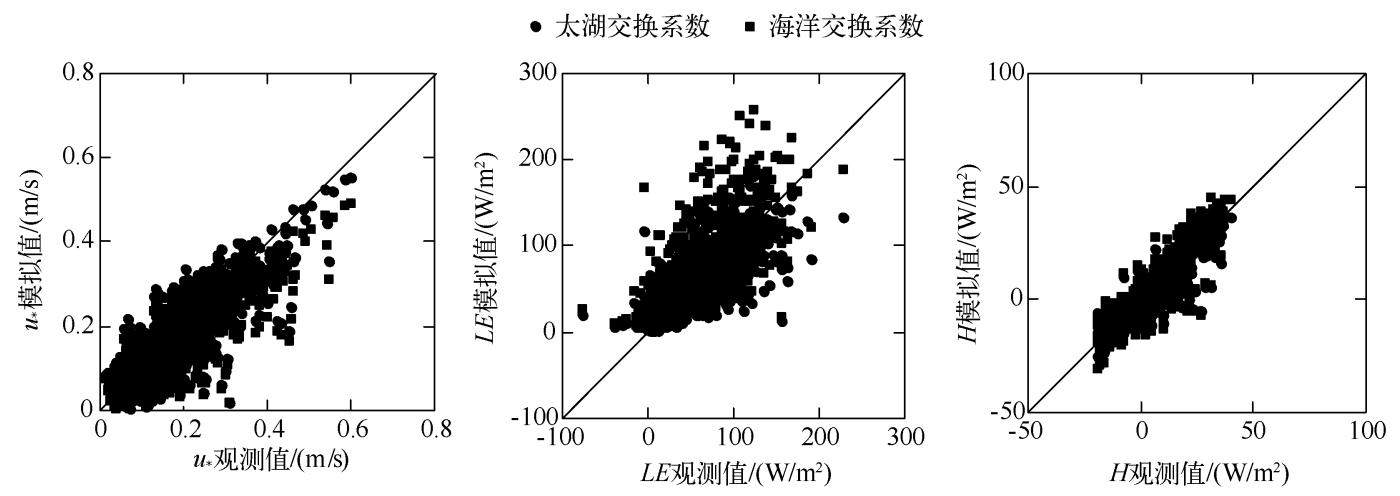

图 1 TLLER 站 $u_{*} 、 H$ 和 $L E$ 的观测值与模拟值 (无稳定度校正) 对比

Fig. 1 Observed versus calculated $u_{*}, H$ and $L E$ at TLLER Station in Lake Taihu (without stability corrections) using two different sets of transfer coefficients

\section{3 敏感性分析}

稳定度校正后的交换系数与中性条件交换系数的关系为 ${ }^{[18]}$ :

$$
\begin{gathered}
C_{\mathrm{D}} / C_{\mathrm{DN}}=\left[1-\Psi_{\mathrm{M}}(\zeta) / \ln \left(z / z_{0}\right)\right]^{-2} \\
C_{\mathrm{E}} / C_{\mathrm{EN}}=\left[1-\Psi_{\mathrm{M}}(\zeta) / \ln \left(z / z_{0}\right)\right]^{-1}\left[1-\Psi_{\mathrm{E}}(\zeta) / \ln \left(z / z_{\mathrm{q}}\right)\right]^{-1} \\
C_{\mathrm{H}} / C_{\mathrm{HN}}=\left[1-\Psi_{\mathrm{M}}(\zeta) / \ln \left(z / z_{0}\right)\right]^{-1}\left[1-\Psi_{\mathrm{H}}(\zeta) / \ln \left(z / z_{\mathrm{T}}\right)\right]^{-1}
\end{gathered}
$$

式中, $\zeta$ 为 Obukhov 稳定度参数, $\Psi_{\mathrm{M}} 、 \Psi_{\mathrm{E}}$ 和 $\Psi_{\mathrm{H}}$ 为稳定度校正函数. 

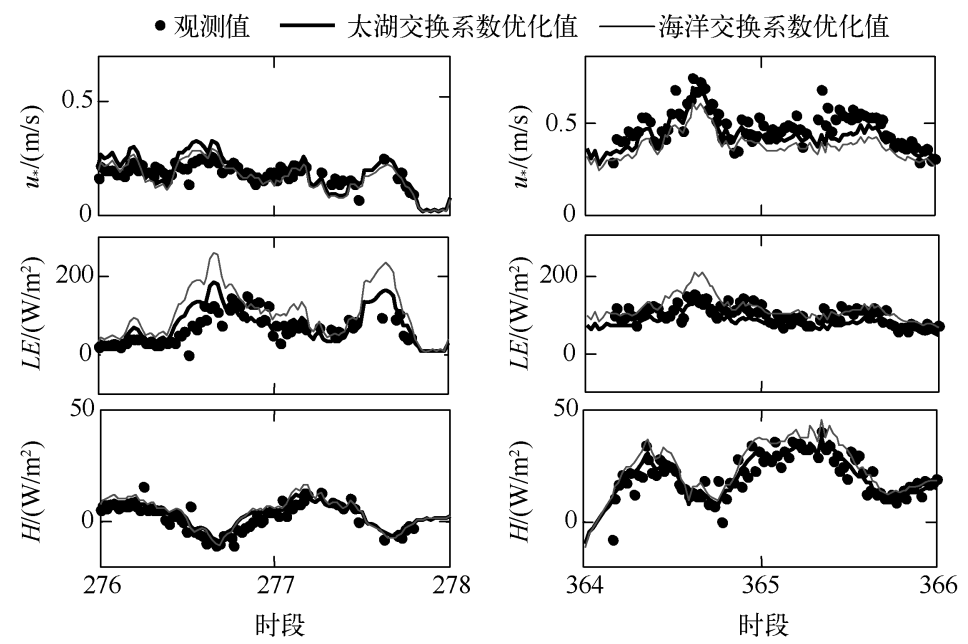

图 2 太湖 TLLER 站 $u_{*} 、 H$ 和 $L E$ 的观测值和计算值

(图中选择的时段是风浪吹程足够大并且仪器受支架影响最小的风向范围)

Fig. 2 Time series of observed and calculated $u_{*}, H$ and $L E$ at TLLER Station

in Lake Taihu (During these two periods, the wind direction satisfied our data screen criteria for fetch and minimal aerodynamic interference by the instrument mounting hardware)

上述结果未进行稳定度校正, 如果用稳定度校正后的交换系数计算潜热通量和感热通量, 其中 $L E$ 的 $R M S E$ 降低了 $1.2 \mathrm{~W} / \mathrm{m}^{2}, M E$ 降低了 $0.5 \mathrm{~W} / \mathrm{m}^{2} ; H$ 的 $R M S E$ 和 $M E$ 降低了 $0.1 \mathrm{~W} / \mathrm{m}^{2}$ 和 $0.4 \mathrm{~W} / \mathrm{m}^{2} ; u_{*}$ 的计算 值没有变化 (表 3). 若在海洋参数化方案中考虑稳定度校正, 改善也不大. 这一结果是合理的, 因为 $83 \%$ 的 数据为近中性条件 $(|\zeta|<0.1)$.

\section{表 3 通量计算结果 *}

Tab. 3 The results of fluxes calculation

\begin{tabular}{|c|c|c|c|c|c|}
\hline \multirow[b]{2}{*}{ 参数化方案 } & \multirow[b]{2}{*}{ 通量 } & \multicolumn{2}{|c|}{ 无稳定度校正 } & \multicolumn{2}{|c|}{ 有稳定度校正 } \\
\hline & & $\begin{array}{c}\text { 均方根误差 } \\
(\text { RMSE })\end{array}$ & $\begin{array}{c}\text { 平均误差 } \\
(M E)\end{array}$ & $\begin{array}{c}\text { 均方根误差 } \\
(\text { RMSE })\end{array}$ & $\begin{array}{c}\text { 平均误差 } \\
(M E)\end{array}$ \\
\hline \multirow[t]{3}{*}{ 太湖 } & $u_{*} /(\mathrm{m} / \mathrm{s})$ & 0.062 & -0.011 & 0.061 & -0.011 \\
\hline & $L E /\left(\mathrm{W} / \mathrm{m}^{2}\right)$ & 26.6 & -2.1 & 25.4 & -1.6 \\
\hline & $H /\left(\mathrm{W} / \mathrm{m}^{2}\right)$ & 5.5 & -1.1 & 5.4 & -0.7 \\
\hline \multirow[t]{3}{*}{ 海洋参数化方案结果 } & $u_{*} /(\mathrm{m} / \mathrm{s})$ & 0.066 & -0.032 & 0.066 & -0.031 \\
\hline & $L E /\left(\mathrm{W} / \mathrm{m}^{2}\right)$ & 40.6 & 23.1 & 41.5 & 25.6 \\
\hline & $H /\left(\mathrm{W} / \mathrm{m}^{2}\right)$ & 5.9 & -0.1 & 6.1 & 0.6 \\
\hline \multirow[t]{3}{*}{ COARE 模型交换系数常值 } & $u_{*} /(\mathrm{m} / \mathrm{s})$ & 0.071 & -0.040 & 0.070 & -0.040 \\
\hline & $L E /\left(\mathrm{W} / \mathrm{m}^{2}\right)$ & 28.5 & 6.3 & 27.9 & 7.9 \\
\hline & $H /\left(\mathrm{W} / \mathrm{m}^{2}\right)$ & 5.6 & -1.5 & 5.4 & -1.0 \\
\hline \multirow[t]{3}{*}{ COARE 模型与风速相关的交换系数 } & $u_{*} /(\mathrm{m} / \mathrm{s})$ & 0.064 & -0.036 & 0.064 & -0.036 \\
\hline & $L E /\left(\mathrm{W} / \mathrm{m}^{2}\right)$ & 29.8 & 7.6 & 29.2 & 9.2 \\
\hline & $H /\left(\mathrm{W} / \mathrm{m}^{2}\right)$ & 5.6 & -1.1 & 5.5 & -0.7 \\
\hline
\end{tabular}

* 太湖的交换系数为: $C_{\mathrm{D} 10 \mathrm{~N}}=1.52 \times 10^{-3} 、 C_{\mathrm{E} 10 \mathrm{~N}}=0.82 \times 10^{-3} 、 C_{\mathrm{H} 10 \mathrm{~N}}=1.02 \times 10^{-3}$; 海洋参数化方案结果为: $C_{\mathrm{D} 10 \mathrm{~N}}=$ $1.21 \times 10^{-3} 、 C_{\mathrm{E} 10 \mathrm{~N}}=1.14 \times 10^{-3} 、 C_{\mathrm{H} 10 \mathrm{~N}}=1.22 \times 10^{-3}$; $\mathrm{COARE}$ 模式的交换系数为: $C_{\mathrm{D} 10 \mathrm{~N}}=1.08 \times 10^{-3} 、 C_{\mathrm{E} 10 \mathrm{~N}}=0.90 \times$ $10^{-3} 、 C_{\mathrm{H} 10 \mathrm{~N}}=0.96 \times 10^{-3}$. 
海洋大气近地层的交换系数通常为风速的函数 (附录 1). 我们采用 COARE 模式进行稳定度分析, 以太 湖的风速和摩擦速度观测值为输人变量, 计算得到的 $C_{\mathrm{D} 10 \mathrm{~N}} 、 C_{\mathrm{E} 1 \mathrm{~N} \mathrm{~N}}$ 和 $C_{\mathrm{H} 10 \mathrm{~N}}$ 变化范围为 $0.97 \times 10^{-3} \sim 1.63 \times$ $10^{-3} 、 0.91 \times 10^{-3} \sim 1.16 \times 10^{-3}$ 和 $0.91 \times 10^{-3} \sim 1.16 \times 10^{-3}$, 平均值为 $1.08 \times 10^{-3} 、 0.96 \times 10^{-3}$ 和 $0.96 \times$ $10^{-3}$ (表 2). 如果采用这些交换系数的平均值计算通量, 不如太湖交换系数的结果好, $u_{*} 、 L E$ 和 $H$ 的 RMSE 和 $M E$ 分别为 0.071 和 $-0.040 \mathrm{~m} / \mathrm{s} 、 28.5$ 和 $6.3 \mathrm{~W} / \mathrm{m}^{2} 、 5.6$ 和 $-1.5 \mathrm{~W} / \mathrm{m}^{2}$. 如果考虑风速的影响, $u$ *的模拟 值有所改善, $R M S E$ 和 $M E$ 分别降低 0.007 和 $0.004 \mathrm{~m} / \mathrm{s} ; L E$ 的模拟效果变差, $R M S E$ 和 $M E$ 都升高了 $1.3 \mathrm{~W} / \mathrm{m}^{2} ; H$ 的模拟结果几乎不受影响.

\section{3 结论}

根据太湖的涡度相关观测资料, 本文得到交换系数的优化值为 $C_{\mathrm{D} 10 \mathrm{~N}}=1.52 \times 10^{-3} 、 C_{\mathrm{E} 10 \mathrm{~N}}=0.82 \times 10^{-3}$ 和 $C_{\mathrm{H} 10 \mathrm{~N}}=1.02 \times 10^{-3}$. 与文献中另外 4 个内陆湖泊的值相近. 所有这 5 个湖泊的交换系数平均值为 $C_{\mathrm{D} 10 \mathrm{~N}}=$ $1.54 \times 10^{-3} 、 C_{\mathrm{E} 10 \mathrm{~N}}=1.03 \times 10^{-3}$ 和 $C_{\mathrm{H} 10 \mathrm{~N}}=1.02 \times 10^{-3}$.

本文用太湖的观测资料评价了 9 个海洋参数化方案在浅水湖泊中的适用性. 在太湖试验的风速条件 下, 海洋参数化方案计算的交换系数平均值为 $C_{\mathrm{D} 10 \mathrm{~N}}=(1.21 \pm 0.14) \times 10^{-3} 、 C_{\mathrm{E} 10 \mathrm{~N}}=(1.14 \pm 0.11) \times 10^{-3}$ 和 $C_{\mathrm{H} 10 \mathrm{~N}}=(1.22 \pm 0.39) \times 10^{-3}$. 结果表明相同的风速条件下, 浅水湖泊拖曳系数的优化值高于海洋参数化方 案的结果. 如果用海洋参数化方案的结果估算湖泊年蒸发量, 偏差可达 $40 \%$.

本文在确定交换系数时忽略了稳定度和风速的影响. 敏感性分析结果表明, 考虑稳定度校正和风速的 影响,并没有改善通量的计算结果,因此这一简化是合理的.

\section{附录 1 海洋交换系数参数化方案}

本附录总结了 9 个常用海气通量的参数化方案. 其中一些参数化方案直接计算海表粗䊅度, 可以根据 公式( $7 \sim 9$ ) 转换成交换系数 $C_{\mathrm{D} 10 \mathrm{~N}} 、 C_{\mathrm{E} 10 \mathrm{~N}}$ 和 $C_{\mathrm{H} 10 \mathrm{~N}}$.

在 Bourras-Dupuis-Yelland 通量算法 (BDY-NC) ${ }^{[37-38]}$ 中, 低风速和高风速中性稳定度条件下的交换系数 采用的计算方法不同 ${ }^{[39-40]}$. 低风速条件下, 交换系数根据 SOFIA (Surface of the Oceans, Fluxes and Interaction with the Atmosphere) 和 SEMAPHORE (Structure des Echanges Mer-Atmosphere, Properties des Heterogeneites Oceaniques Recherche Experimentale) 观测试验 ${ }^{[39]}$ 确定:

$$
\begin{gathered}
C_{\mathrm{D} 10 \mathrm{~N}}=0.0117 u_{10}^{-2}+0.000668, u_{10} \leqslant 5.85 \mathrm{~m} / \mathrm{s} \\
C_{\mathrm{E} 1 \mathrm{~N}}=\frac{0.00279}{u_{10}}+0.000658, u_{10} \leqslant 5.2 \mathrm{~m} / \mathrm{s}
\end{gathered}
$$

高风速条件下 ${ }^{[40]}:$

$$
\begin{aligned}
C_{\mathrm{D} 10 \mathrm{~N}} & =0.00007 u_{10}+0.0006, u_{10}>5.85 \mathrm{~m} / \mathrm{s} \\
C_{\mathrm{EION}} & =\frac{0.00279}{5.2}+0.000658, u_{10}>5.2 \mathrm{~m} / \mathrm{s}
\end{aligned}
$$

而所有风速条件下:

$$
C_{\text {HoN }}=0.0007
$$

在美国国家大气研究中心大气模式 (第 3 版, CAM3 $)^{[22]}$ 中, 海面的动量交换系数 ${ }^{[57]}$ 为:

$$
C_{\mathrm{D} 10 \mathrm{~N}}=\frac{0.0027}{u_{10}}+0.000142+0.0000764 u_{10}
$$

而 $C_{\mathrm{E} 10 \mathrm{~N}}$ 和 $C_{\mathrm{H} 10 \mathrm{~N}}$ 由粗粘度计算得到 $z_{\mathrm{q}}=9.5 \times 10^{-5} \mathrm{~m}$; 稳定条件下 $z_{\mathrm{T}}=2.2 \times 10^{-9} \mathrm{~m}$, 不稳定条件下 $z_{\mathrm{T}}=$ $4.9 \times 10^{-5} \mathrm{~m}^{[42]}$.

海洋大气耦合响应实验通量算法 (COARE 3.0 $)^{[58]}$ 的算法考虑重力波的作用, Charnock 系数 $a$ 与风速的 
关系为：

$$
a= \begin{cases}0.011 & u_{10} \leqslant 10 \mathrm{~m} / \mathrm{s} \\ 0.011+\frac{0.007}{8}\left(u_{10}-10\right) & 10<u_{10}<18 \mathrm{~m} / \mathrm{s} \\ 0.018 & u_{10} \geqslant 18 \mathrm{~m} / \mathrm{s}\end{cases}
$$

粗䊁度为:

$$
\begin{gathered}
z_{0}=\frac{a u_{*}^{2}}{g}+\frac{0.11 v}{u_{*}} \\
z_{\mathrm{T}}=z_{\mathrm{q}}=\min \left(1.1 \times 10^{-5} \mathrm{~m}, 5.5 \times 10^{-5} R_{*}^{-0.6}\right)
\end{gathered}
$$

式中, $R e_{*}$ 是粗糙雷诺数, $R e_{*}=z_{0} u_{*} / v, v$ 是空气动力粘滞系数, $g$ 是重力加速度.

欧洲中期天气预报模型 $(\mathrm{ECMWF})^{[4546]}$ 中粗鋉度是 $u_{*}$ 的函数:

$$
\begin{gathered}
z_{0}=\frac{0.018 u_{*}^{2}}{g}+\frac{0.65 \times 10^{-6}}{u_{*}} \\
z_{\mathrm{T}}=\frac{6 \times 10^{-6}}{u_{*}} \\
z_{\mathrm{q}}=\frac{9.3 \times 10^{-6}}{u_{*}}
\end{gathered}
$$

在 Goddard 地球观测系统同化资料 (GEOS-2 $)^{[59]}$ 中动量粗䊅度是摩擦风速的函数:

$$
z_{0}=\frac{A_{1}}{u_{*}}+A_{2}+A_{3} u_{*}+A_{4} u_{*}^{2}+A_{5} u_{*}^{3}
$$

式中, 低风速情况下, 系数 $A_{1} 、 A_{2} 、 A_{3} 、 A_{4}$ 和 $A_{5}$ 取值于文献 [47], 中、高风速条件下, 取值于文献 [41]. 水汽和 热量粗粘度与动量粗粘度的关系是:

$$
\ln \left(\frac{z_{0}}{z_{\mathrm{T}}}\right)=\ln \left(\frac{z_{0}}{z_{\mathrm{q}}}\right)=0.72\left(R e_{*}-0.135\right)^{1 / 2}
$$

在 Goddard 卫星反演地表湍流通量资料(版本 2, GSSTF-2) 中, 动量粗粘度 ${ }^{[48]}$ 为:

$$
z_{0}=\frac{0.0144 u_{*}^{2}}{g}+\frac{0.11 v}{u_{*}}
$$

热量和水汽粗鋉度 ${ }^{[58]}$ 为:

$$
\begin{aligned}
& \frac{z_{\mathrm{T}} u_{*}}{v}=a_{1} R e_{*}^{b_{1}} \\
& \frac{z_{\mathrm{q}} u_{*}}{v}=a_{2} R \boldsymbol{e}_{*}^{b_{2}}
\end{aligned}
$$

式中, $a_{1} 、 a_{2} 、 b_{1}$ 和 $b_{2}$ 的取值见文献 $[58]$.

卫星数据反演的 Hamburg 海气参数 (HOAPS $)^{[49]}$ 中动量粗䊁度为:

$$
z_{0}=\frac{0.011 u_{*}^{2}}{g}+\frac{0.11 v}{u_{*}}
$$

热量和水汽的交换系数为 $C_{\mathrm{H} 10 \mathrm{~N}}=1 \times 10^{-3}$ 和 $C_{\mathrm{E} 10 \mathrm{~N}}=1.2 C_{\mathrm{H} 10 \mathrm{~N}}$.

在日本海洋通量遥感观测数据库产品中 $(\mathrm{J}-\mathrm{OFURO})^{[50]}, 10 \mathrm{~m}$ 高度处的拖曳系数 ${ }^{[42]}$ 为: 


$$
C_{\text {D10N }}=\left\{\begin{array}{l}
1.14 \times 10^{-3}, u_{10}<10 \mathrm{~m} / \mathrm{s} \\
\left(0.49+0.065 \mathrm{u}_{10}\right) \times 10^{-3}, 10<\mathrm{u}_{10}<25 \mathrm{~m} / \mathrm{s}
\end{array}\right.
$$

水汽交换系数 ${ }^{[47]}$ 为:

$$
C_{\mathrm{E} 10 \mathrm{~N}}=a_{\mathrm{e}}+b_{\mathrm{e}} u_{10}^{p_{e}}+c_{\mathrm{e}}\left(u_{10}-8\right)^{2}
$$

式中, 系数 $a_{\mathrm{e}} 、 b_{\mathrm{e}} 、 c_{\mathrm{e}}$ 和 $p_{\mathrm{e}}$ 的取值见文献 $[47] . J-O F U R O$ 中用潜热通量和波文比 $B$ 计算感热通量, 热量交换系 数为:

$$
C_{\mathrm{H} 10 \mathrm{~N}}=\frac{B L_{v} C_{\mathrm{E} 10 \mathrm{~N}}\left(q_{s}-q_{a}\right)}{C_{\mathrm{p}}\left(\theta_{s}-\theta_{a}\right)}
$$

最后, 亚利桑那大学空气动力学算法中计算海洋通量时, 粗䊁度的算法 ${ }^{[20,49,51]}$ 为:

$$
\begin{gathered}
z_{0}=\frac{0.013 u_{*}^{2}}{g}+\frac{0.11 v}{u_{*}} \\
\ln \left(\frac{z_{0}}{z_{\mathrm{T}}}\right)=\ln \left(\frac{z_{0}}{z_{\mathrm{q}}}\right)=2.67 \operatorname{Re}_{*}^{1 / 4}-2.57
\end{gathered}
$$

\section{4 参考文献}

[ 1 ] Downing JA, Prairie YT, Cole JJ et al. The global abundance and size distribution of lakes, ponds, and impoundments. Limnol Oceanogr, $2006, \mathbf{5 1}(5): 2388-2397$.

[ 2 ] Henderson-Sellers B. Calculating the surface energy balance for lake and reservoir modeling: A review. Rev Geophys, 1986,24 (3):625-649.

[ 3 ] Bonan GB. Sensitivity of a GCM simulation to inclusion of inland water surfaces. J Clim, 1995,8(11):2691-2704.

[ 4 ] Betts AK, Ball JH. Albedo over the boreal forest. J Geophys Res, 1997,102(D24) :28901-28909.

[ 5 ] Liu H, Randerson JT, Lindfors J et al. Changes in the surface energy budget after fire in boreal ecosystems of interior Alaska: An annual perspective. J Geophys Res, 2005 ,110(13): D13101 (doi:10. 1029/2004JD005158).

[ 6 ] Rouse WR, Oswald CM, Binyamin J et al. Interannual and seasonal variability of the surface energy balance and temperature of central Great Slave Lake. J Hydrometeorol, 2003,4 (4) : 720-730.

[ 7 ] Rouse WR, Oswald CJ, Binyamin et al. The role of northern lakes in a regional energy balance. J Hydrometeorol, 2005 , 6(3) :291-305 (doi:10.1175/JHM421. 1).

[ 8 ] Long Z, Perrie W, Gyakum J et al. Northern lake impacts on local seasonal climate. J Hydrometeorol, 2007,8(4):881896.

[ 9 ] Samuelsson P, Tjernström M. Mesoscale flow modification induced by land-lake surface temperature and roughness differences. J Geophys Res, 2001 ,106( D12) :12419-12435.

[10] Törnblom K, Bergström H, Johansson C. Thermally driven mesoscale flows-simulations and measurements. Boreal Environ Res, $2007, \mathbf{1 2}(6): 623-641$.

[11] Ljungemyr P, Gustafsson N, Omstedt A. Parameterization of lake thermodynamics in a high-resolution weather forecasting model. Tellus A, 1996,48(5) :608-621.

[12 ] Dutra E, Stepanenko VM, Balsamo G et al. An offline study of the impact of lakes on the performance of the ECMWF surface scheme. Boreal Environ Res, 2010,15(2):100-112.

[13] 中国科学院南京地理与湖泊研究所. 抚仙湖. 北京: 海洋出版社, 1990:59-61.

[14] Elo PAR. The energy balance and vertical thermal structure of two small boreal lakes in summer. Boreal Environ Res, $2007,12(5): 585-600$.

[15] Rouse WR, Blanken PD, Bussières N et al. An investigation of the thermal and energy balance regimes of Great Slave and Great Bear lakes. J Hydrometeorol, 2008 ,9(6) : 1318-1333 (doi :10. 1175/2008JHM977. 1 ).

[16] Liu H, Zhang Y, Liu S et al. Eddy covariance measurements of surface energy budget and evaporation in a cool season over southern open water in Mississippi. J Geophys Res, 2009, 114(4) : D04110 (doi:10. 1029/2008JD010891). 
[17] Dyer AJ. A review of flux-profile relationships. Boundary-Layer Meteorol, 1974,7(3) :363-372.

[18] Garratt JR. The atmospheric boundary layer. Cambridge: Cambridge University Press, 1992.

[19] Grachev AA, Fairall CW. Dependence of the Monin-Obukhov stability parameter on the bulk Richardson number over the ocean. J Appl Meteorol, $1997,36(4): 406-414$.

[20] Zeng X, Zhao M, Dickinson RE. Intercomparison of bulk aerodynamic algorithms for the computation of sea surface fluxes using TOGA COARE and TAO data. J Clim, $1998, \mathbf{1 1}$ ( 10 ) :2628-2644.

[21] Brunke MA, Zeng XB, Anderson S. Uncertainties in sea surface turbulent flux algorithms and data sets. J Geophys Res, $2002, \mathbf{1 0 7}$ (C10) : 3141 (doi : 10. 1029/2001JC000992).

[22] Collins WD, Rasch PJ, Boville BA et al. Description of the NCAR community Atmosphere Model (CAM3). In: Technical report NCAR/TN-464 + STR. Boulder: National Center for Atmospheric Research, 2004.

[23 ] Ban J, Gao Z, Lenschow DH. Climate simulations with a new air-sea turbulent flux parameterization in the National Center for Atmospheric Research Community Atmosphere Model (CAM3). J Geophys Res, 2010,115: D01106 ( doi : 10. 1029/ 2009JD012802).

[24] Li Y, Gao Z, Lenschow DH et al. An improved approach for parameterizing surface-layer turbulent transfer coefficients in numerical models. Boundary-Layer Meteorol, 2010,137 (1) :153-165.

[25] Martínez-Alvarez V, Gallego-Elvira B, Maestre-Valero JF et al. Simultaneous solution for water, heat and salt balances in a Mediterranean coastal lagoon (Mar Menor, Spain). Estuar, Coast \& Shelf Sci, 2011,91 (2) :250-261.

[26] Krinner G. Impact of lakes and wetlands on boreal climate. J Geophys Res, 2003,108 ( D16) : 4520 (doi:10. 1029/ 2002JD002597).

[27] MacKay MD, Neale PJ, Arp CD et al. Modeling lakes and reservoirs in the climate system. Limnol Oceanogr, 2009 , 54 (6, part2):2315-2329.

[28 ] Atakturk SS, Katsaros KB. Wind stress and surface waves observed on Lake Washington. J Phys Oceanogr, 1999,29(4) : $633-650$.

[29] Donelan MA, Dobson FW, Smith SD et al. On the dependence of sea-surface roughness on wave development. J Phys Oceanogr, $1993,23(9): 2143-2149$.

[30] Gao ZQ, Wang Q, Zhou MY. Wave-dependence of friction velocity, roughness length, and drag coefficient over coastal and open water surfaces by using three databases. Adv Atmos Sci, 2009,26(5) :887-894.

[31] Geernaert GL, Larsen SE, Hansen F. Measurements of the wind-stress, heat-flux andturbulence intensity during storm conditions over the north sea. J Geophys Res, 1987,92( C12) :13127-13139.

[32] Panin GN, Nasonove AE, Foken Th et al. On the parameterisation of evaporation and sensible heat exchange for shallow lakes. Theor Appl Climatol, 2006,85(3/4):123-129.

[33 ] Smith SD, Anderson RJ, Oost WA et al. Sea-surface wind stress and drag coefficients — the HEXOS results. BoundaryLayer Meteorol, $1992, \mathbf{6 0}(1 / 2): 109-142$.

[34] Heikinheimo M, Kangas M, Tourula T et al. Momentum and heat fluxes over lakes Tämnaren and Råksjö determined by the bulk-aerodynamic and eddy-correlation methods. Agric For Meteorol, 1999,98/99:521-534.

[35] Blanken PD, Rouse WR, Schertzer WM. Enhancement of evaporation from a large northern lake by the entrainment of warm, dry air. J Hydrometeorol, 2003,4 (4):680-693.

[36] Nordbo A, Launiainen S, Mammarella I et al. Long-term energy flux measurements and energy balance over a small boreal lake using eddy covariance technique. J Geophys Res, 2011,116:D02119( doi :10. 1029/2010JD014542).

[37] Bourras D. Estimation of the latent heat flux over oceans from satellite data [Dissertation ]. Paris: Paris VI University, 1999.

[38] Bourras D. Calcul des flux turbulents au dessus des océans par la méthode bulk. (Calculation of turbulent fluxes over oceans using the bulk method) Centre d'Etude des Environnements Terrestre et Planétaires Tech. Note RI_CETP/2/ 2000, 2000 : 54. [Availabel from CETP, 10-12 av. De l'Europe, 78140, Velizy, France. ]

[39] Dupuis H, Taylor PK, Weill A et al. Inertial dissipation method applied to derive turbulent fluxes over the ocean during the surface of the ocean, fluxes and interactions with the atmosphere/atlantic stratocumulus transition experiment (SOFIA/ ASTEX) and structure des echanges mer-Atmosphere, proprietes des heterogeneites oceaniques: recherche experimentale (SEMAPHORE) experiments with low to moderate wind speeds. J Geophys Res, 1997,102 (C9) :21115-21129. 
[40 ] Yelland M, Taylor PK. Wind stress measurements from the open ocean. J Phys Oceanogr, 1996,26(4) :541-558.

[41] Large WG, Pond S. Open ocean momentum flux measurements in moderate to strong winds. J Phys Oceanogr $, 1981,11$ (3) :324-336.

[42] Large WG, Pond S. Sensible and latent heat flux measurements over the ocean. J Phys Oceanogr, 1982,12(5):464-482.

[43] Fairall CW, Bradley EF, Hare JE et al. Bulk parameterization on air-sea fluxes: Updates and verification for the COARE algorithm. J Clim, 2003,16(4) :571-591.

[44] Fairall CW, Bradley EF, Rogers DP et al. Bulk parameterization of air-sea fluxes for Trophical Ocean-Global Atmosphere Coupled-Ocean Atmosphere Response Experiment. J Geophys Res, 1996,101( C2 ) :3747-3764.

[45] Beljaars ACM. The impact of some aspects of the boundary layer scheme in the ECMWF model. In Seminar Proceedings on Parametrization of Sub-grid Scale Physical Processes. Shinfield Park: ECMWF, 1995 :125-162.

[46] Beljaars ACM. The parameterization of surface fluxes in large scale models under free convection. Quarterly Journal of the Royal Meteorological Society, 1995,121 (522):255-270.

[47] Kondo J. Air-sea bulk transfer coefficients in diabatic conditions. Boundary-Layer Meteorol, 1975,9(1):91-112.

[48] Chou S. A comparison of airborne eddy correlation and bulk aerodynamic methods for ocean-air turbulent fluxes during cold-air outbreaks. Boundary-Layer Meteorol, $1993,64(1): 75-100$.

[49] Smith SD. Coefficients for sea surface wind stress, heat flux, and wind profiles as a function of wind speed and temperature. J Geophys Res, 1988,93(C12) :15467-15472.

[50] Kubota M, Mitsumori S. Sensible heat flux estimated by satellite data over the North Pacific. In: Liu CT ed. Remote sensing of subtropical oceans. New York: Elsevier, 1997:127-136.

[51] Brutsaert WH. Evaporation into the atmosphere: Theory, history, and applications. Boston: P. Reidel Publishing Company, 1982.

[52] Brunke MA, Fairall CW, Zeng X et al. Which bulk aerodynamic algorithms are least problematic in computing ocean surface turbulent fluxes? J Clim, 2003 ,16(4):619-635.

[53] 毛 锐. 太湖、团氿湖水面蒸发的初步研究. 海洋与湖沼, 1978,9(1):26-35.

[54] 毛新伟,高 怡,徐卫东. 水文巡测方法对太湖水量平衡计算的影响. 水文,2006,26(5):58-60.

[55] 申金玉,石亚东,高 怡等.太湖水量平衡影响因素分析及误差控制措施研究. 水文,2011,31(3):60-63.

[56] Yu L, Weller RA. Objectively analyzed air-sea heat fluxes for the global ice-free oceans (1981-2005). Bull Am Meteorol Soc, $2007, \mathbf{8 8}(4): 527-539$.

[57] Large WG, McWilliams JC, Doney SC. Oceanic vertical mixing: A review and a model with a nonlocal boundary layer parameterization. Rev Geophys, 1994,32 (4) :363-403.

[58 ] Liu WT, Katsaros KB, Businger JA. Bulk parameterization of air-sea exchanges of heat and water vapor including the molecular constraints at the interface. J Atmos Sci, 1979,36(9):1722-1735.

[59] Data Assimilation Office (DAO). Algorithm theoretical basis document for Goddard Earth Observing System Data Assimilation System ( GEOS DAS) with a focus on version 2. Data Assimilation Office, NASA Goddard Space Flight Center, 1996. 